\title{
Log-concavity of the genus polynomials for a sequence of cubic Halin graphs
}

\author{
Jonathan L. Gross, Toufik Mansour, and Thomas W. Tucker
}

\begin{abstract}
A Halin graph is a graph obtained from a plane tree by running a cycle through its leaf vertices in the order they are encountered along a counterclockwise pre-order traversal. Using a vectorized production matrix, we give a matrix formula for the partitioned genus polynomial of any cubic Halin graph and for the genus polynomial as well. We prove log-concavity of the genus polynomial and of the partitioned genus polynomials for several sequences of cubic Halin graphs, which serves as further support of the conjecture that the genus polynomial of every graph is log-concave.
\end{abstract}

Our general concern is enumerating all possible cellular imbeddings $f: G \rightarrow S$ of a given graph $G$ in an oriented surface $S$. An imbedding is cellular if the interior of every face (component of $S-f(G)$ ) is homeomorphic to an open disk. Two imbeddings $f: G \rightarrow S$ and $g: G \rightarrow S$ are regarded as the same if there is an orientation-preserving homeomorphism of the surface $S$ taking $f(G)$ to $g(G)$ that induces the identity automorphism of $G$.

Our enumeration of imbeddings is according to the genus of the imbedding surface. We define the genus distribution of a finite graph $G$ to be the sequence

$$
\left\langle g_{i}(G) \mid i=0,1,2, \ldots\right\rangle
$$

where $g_{i}(G)$ counts the cellular imbeddings of $G$ in the closed orientable surface $S_{i}$ of genus $i$. The genus polynomial $g_{G}(z)$ of $G$ is the generating function $\Sigma g_{i}(G) z^{i}$.

To understand the derivation of genus distributions, we must understand how adding an edge to a graph $G$ affects the genus distribution and its properties. Adding an edge between two 2-valent vertices with a common neighbor is one of the simplest versions of this situation. In this paper, we are within a context for this kind of edge-adding in which the graph $G$ to be modified is from a family of known genus distribution. One way of looking at the two other families of Halin graphs for which we derive genus polynomials and prove their log-concavity, beyond the family of Halin graphs that are 


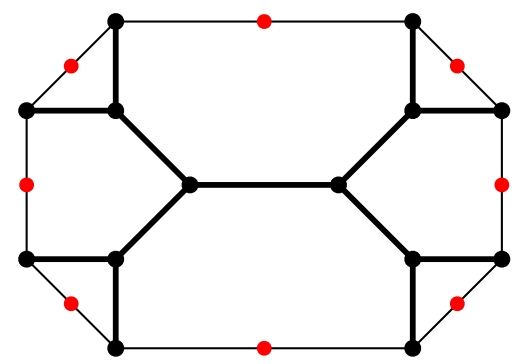

Figure 1: Halin graph plus red midpoints on the exterior cycle.

isomorphic to Ringel ladders, is that each of the graphs in these two other families is obtained by adding a single edge to a Ringel ladder.

Section 1 reviews the quadrangulation of plane cubic Halin graphs, from [2]. Section 2 shows how they fit with the subsequently invented construct [6] called a vectorized production matrix. In Section 3, we prove that the cubic Halin graphs can be counted with Catalan numbers. The genus polynomials of some infinite sequences of Halin graphs are derived in Section 4. They are proved to be log-concave in Section 5. Moreover, we show that the partitioned genus polynomials of the graphs in that sequence are log-concave, which supports a secondary log-concavity conjecture. In Section 6 , we give a few research problems concerned with extending these results.

\section{Quadrangulation of cubic Halin graphs}

An algorithm is given by [2] for calculating the genus distribution of a cubic Halin graph $G$, which is specified as the 1-skeleton of a 2-complex $K$ on the plane disk $D$ bounded by the outer cycle of $G$. We construe the graph $G$ to be colored black. We give a 3-step process for constructing a 3-colorable quadrangulation of the 2-complex $K$.

Step 1. In each cycle edge of the Halin graph, insert a red midpoint. This is illustrated in Figure 1.

Step 2. Join each red vertex $v$ to all of the non-leaf vertices on the boundary of the face on whose boundary $v$ lies, as shown in Figure 2.

Proposition 1.1. The red and black edges together triangulate the plane disk $D$ bounded by the exterior cycle of a plane Halin graph $G$.

Proof. This is Proposition 3.1 of [2].

Proposition 1.2. Every black tree edge lies on two of the triangles formed by Steps 1 and 2.

Proof. This is Proposition 3.2 of [2]. 


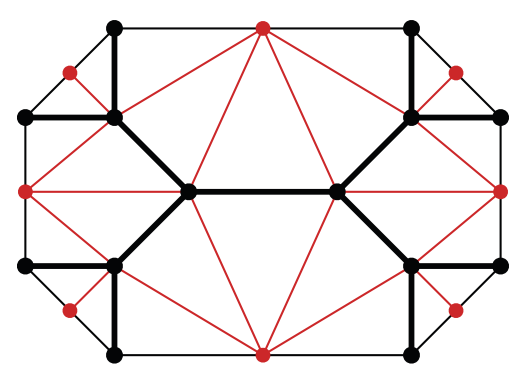

Figure 2: Halin graph plus all of the red edges.

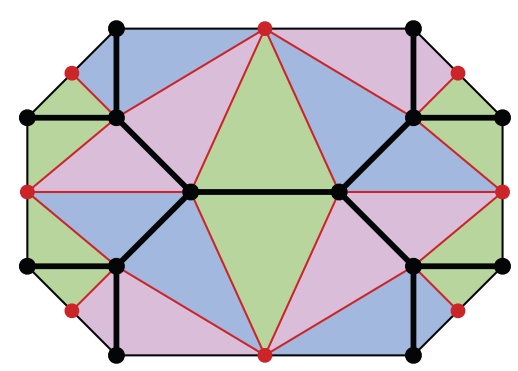

Figure 3: Quadrangulation of a plane Halin graph.

Step 3a. For each black tree edge, we pair the two incident triangles into a quadrangle.

Step 3b. We assign (unseen) colors blue, green, and pink to the tree edges, so as to form a proper edge 3 -coloring. This is possible because any tree of maximum degree 3 is edge-3-colorable (via greedy algorithm).

Step 3c. We visibly color each quadrangle with the unseen color of the tree edge that bisects it, as shown in Figure 3.

The graph that results from deleting all the vertices on the outer cycle and all the edges incident on them from a plane Halin graph $G$ is called the inner tree of $G$.

\section{VP-matrix for the $\pi$-merge operation}

In this section, we reinterpret the genus distribution algorithm of [2] for a cubic Halin graph, in terms of a vectorized production matrix (abbr. vp matrix), a new construct introduced by [6]. Some familiarity with partitioned genus distributions and productions, which are constructs introduced 

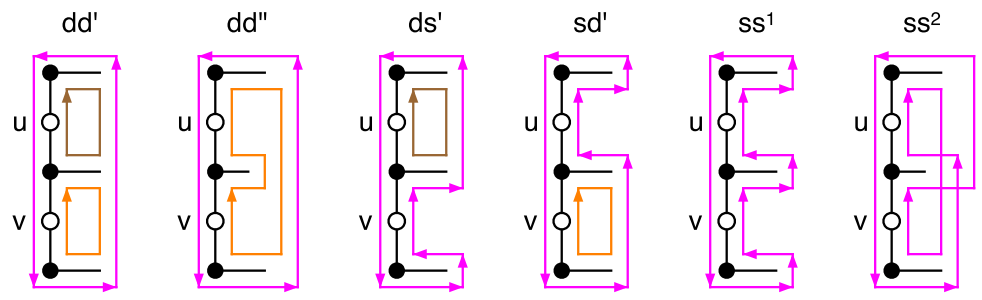

Figure 4: The six double-rooted partials for a $\pi$-merge.

in [4], [9], [8], and [1], is assumed. (A completely general version of these constructs is developed in [3].)

\subsection{Partials for cubic Halin graphs}

For a doubly vertex-rooted cubic Halin graph $(G, u, v)$, with the roots $u$ and $v$ inserted at the midpoints of adjacent edges, we split $g_{i}(G)$ into six partials. Here is what they count:

$d d_{i}^{\prime}$ Each of the roots $u$ and $v$ lies on two distinct fb-walks. One and only one of these fb-walks traverses both roots.

$d d_{i}^{\prime \prime}$ Each of the roots $u$ and $v$ lies on two distinct fb-walks. Both of these fb-walks traverse both roots.

$d s_{i}^{\prime}$ Root $u$ lies on two distinct fb-walks. One of these fb-walks traverses root $v$ twice.

$s d^{\prime}$ Root $v$ lies on two distinct fb-walks. One of these fb-walks traverses root $u$ twice.

$s s_{i}^{1}$ A single fb-walks traverses roots $u$ and $v$ twice. The occurrences of each root are consecutive.

$s s_{i}^{2}$ A single $\mathrm{fb}$-walks traverses roots $u$ and $v$ twice. The occurrences of the two roots alternate.

These configurations are illustrated in Figure 4.

\subsection{Productions for a $\pi$-merge}

We regard the quadrangles of our decomposition of the 2-complex $K$ as atomic fragments, to be reassembled into $K$ itself. During the reassembly, we calculate the partitioned genus distributions of the larger fragments that we construct by iterative mergers, until we have obtained the genus distribution of the entire Halin graph $G$. 

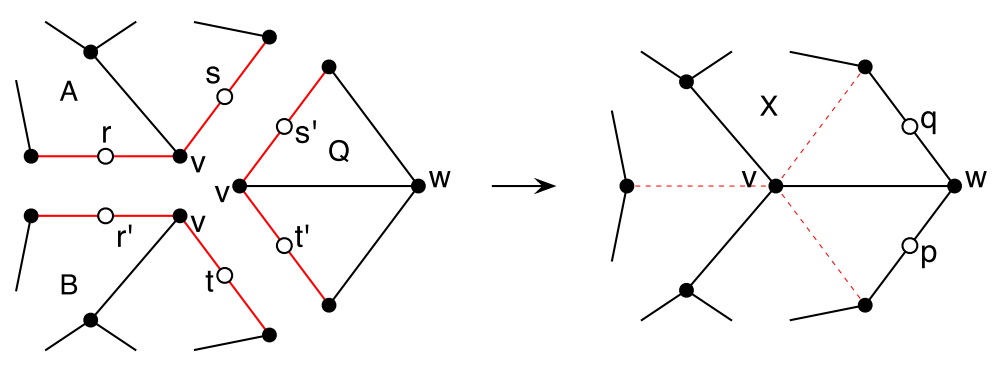

Figure 5: A $\pi$-merge $\left((A, r, s),\left(B, t, r^{\prime}\right),\left(Q, s^{\prime}, t^{\prime}, p, q\right)\right) \rightarrow(X, p, q)$ at vertex $v$.

We merge three fragments at a time, always ordered so that the third fragment is a designated quadrangle $Q \cong K_{4}-e$ with four root-vertices. Envisioning this configuration as a small pie cut into three slices, we call such a 3 -fragment merger a $\pi$-merge,

$$
\left((A, r, s),\left(B, t, r^{\prime}\right),\left(Q, s^{\prime}, t^{\prime}, p, q\right)\right) \longrightarrow(X, p, q),
$$

as illustrated in Figure 5.

We observe the following properties of a $\pi$-merge operation (1).

1. The fragments $(A, r, s),\left(B, t, r^{\prime}\right)$, and $\left(Q, s^{\prime}, t^{\prime}, p, q\right)$ are arranged counterclockwise.

2. The root-mergers are $r * r^{\prime}, s * s^{\prime}$, and $t * t^{\prime}$.

3. The vertices $p, w$, and $q$ lie in counterclockwise order on the resulting fragment $(X, p, q)$.

\subsection{Vectorized production matrices}

When calculating the partitioned genus distribution of a recursively specified sequence of graphs

$$
G_{1}, G_{2}, G_{3}, \ldots
$$

by iterative application of a fixed graph operation, one can either apply a list of productions representing the effect of that graph operation, or one can combine the productions into an ordinary production matrix, whose application to the pgd-vector of the graph $G_{n}$ yields the pgd-vector of the graph $G_{n+1}$. Assuming that the components of the pgd-vector are polynomials in an indeterminate $z$, the components of the (ordinary) production matrix are polynomials in $z$. 
By way of contrast, a graph operation $f$ that combines two graphs $G$ and $H$ into a graph $J$ can be represented by a vectorized production matrix (abbr. $\boldsymbol{v p}$-matrix) $M_{f}$ that is applied to the respective pgd-vectors $X_{G}$ and $X_{H}$ so that

$$
X_{G} M_{f} X_{H}^{t}=X_{J}
$$

The algebraic objects here have the following properties:

1. $X_{G}$ and $X_{H}$ are row-vectors of polynomials in $z$.

2. Each entry of the matrix $M_{f}$ is a row-vector of polynomials in $z$.

3. $X_{J}$ is a row-vector of polynomials in $z$.

Theorem 2.1. The $\pi$-merge operation of [2] corresponds to the following vectorized production matrix:

$$
\begin{aligned}
& \mathbf{M}_{\pi}=\frac{1}{4} \times \\
& \begin{array}{c|cccccc} 
& d d^{\prime} & d d^{\prime \prime} & d s^{\prime} & s d^{\prime} & s s^{1} & s s^{2} \\
\cline { 2 - 6 } d d^{\prime} & d d^{\prime}+2 z d d^{\prime \prime}+z s s^{2} & 2 d d^{\prime}+2 z s s^{2} & 2 d d^{\prime}+2 z s s^{2} & 2 s d^{\prime}+2 z s s^{1} & 4 s d^{\prime} & 2 d s^{\prime}+2 s d^{\prime} \\
d d^{\prime \prime} & 2 d d^{\prime}+2 z s s^{2} & 4 d d^{\prime \prime} & 4 d s^{\prime} & 4 s d^{\prime} & 4 s s^{1} & 2 z^{-1} d d^{\prime}+2 s s^{2} \\
d s^{\prime} & 2 d s^{\prime}+2 z s s^{1} & 4 d s^{\prime} & 4 d s^{\prime} & 4 s s^{1} & 4 s s^{1} & 2 z^{-1} d s^{\prime}+2 s s^{1} \\
s d^{\prime} & 2 d d^{\prime}+2 z s s^{2} & 4 s d^{\prime} & 2 z^{-1} d d^{\prime}+2 s s^{2} & 4 s d^{\prime} & 4 z^{-1} s d^{\prime} & 2 z^{-1} d d^{\prime}+2 s s^{2} \\
s s^{1} & 4 d s^{\prime} & 4 s s^{1} & 4 z^{-1} d s^{\prime} & 4 s s^{1} & 4 z^{-1} s s^{1} & 4 z^{-1} d s^{\prime} \\
s s^{2} & 2 d s^{\prime}+2 s d^{\prime} & 2 z^{-1} d d^{\prime}+2 s s^{2} & 2 z^{-1} d d^{\prime}+2 s s^{2} & 2 z^{-1} s d^{\prime}+2 s s^{1} & 4 z^{-1} s d^{\prime} & z^{-2} d d^{\prime}+2 z^{-1} d d^{\prime \prime}+z^{-1} s s^{2}
\end{array}
\end{aligned}
$$

Proof. This follows from Theorem 4.3 of [2], where all 36 productions are listed. Theorem 4.3 of [2] lists the productions for all $36(=6 \times 6)$ ordered pairs of partials.

In the vp-matrix $M_{\pi}$, each entry has the same algebraic type as the pgd-vectors to which it is to be applied. For a $\pi$-merge, each of the six coordinates corresponds to the partial genus polynomials for that category. We now define six elementary pgd-vectors:

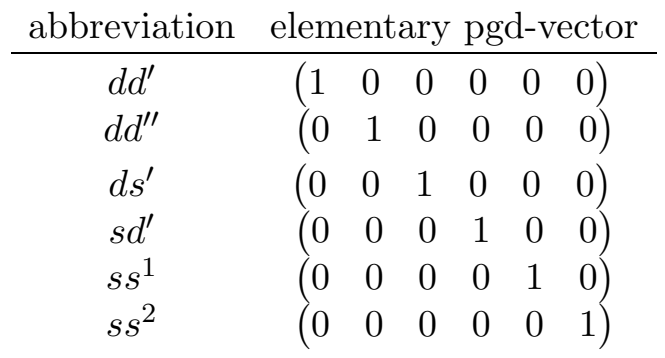


Thus, here are the meanings of some entries for matrix $M_{\pi}$ above.

\begin{tabular}{|c|c|c|}
\hline entry & abbreviation & pgd-vector \\
\hline$M_{f}[1,1]$ & $d d^{\prime}+2 z d d^{\prime \prime}+z s s^{2}$ & $\left(\begin{array}{llllll}1 & 2 z & 0 & 0 & 0 & z\end{array}\right)$ \\
\hline$M_{f}[6,2]$ & $2 z^{-1} d d^{\prime}+2 s s^{2}$ & $\left(\begin{array}{llllll}2 z^{-1} & 1 & 0 & 0 & 0 & 2\end{array}\right)$ \\
\hline$M_{f}[6,6]$ & $z^{-2} d d^{\prime}+2 z^{-1} d d^{\prime \prime}+z^{-1} s s^{2}$ & $\left(\begin{array}{llllll}z^{-2} & 2 z^{-1} & 0 & 0 & 0 & z^{-1}\end{array}\right)$ \\
\hline
\end{tabular}

In what follows, we abbreviate the pgd-vector of a quadrangular fragment as

$$
A=\left[\begin{array}{llllll}
2 & 0 & 0 & 0 & 0 & 2 z
\end{array}\right] .
$$

We abbreviate the matrix $M_{\pi}$ as $M$. When we first $\pi$-merge three quadrangles at vertex $z$ into a 3 -wheel, the combined fragment has the pgd-vector

$$
Y=A M A^{t}=\left[\begin{array}{llllll}
2 & 4 z & 4 z & 4 z & 0 & 2 z
\end{array}\right] .
$$

REMARK. In general, when we apply the $\pi$-merge operation, we mention two fragments (the two explicit operands) in counterclockwise order. The third fragment must always be a quadrangle. The virtual roots always lie on the boundary of whatever quadrangle is the third fragment of the $\pi$-merge.

\section{Pgd-vectors of cubic Halin graphs}

In this section, we establish a bijective correspondence between cubic Halin graphs and rooted binary trees. We also derive the matrix formula for calculating the partitioned genus polynomials and the genus polynomial of any cubic Halin graph. A quadrangulated cubic Halin graph is shown in Figure 6.

The five thicker edges in Figure 6 and the vertices at which they are incident form the inner tree. The pentagonal vertex on the outer cycle is called the anchor. The two hollow vertices on the outer cycle are the virtual roots. The vertex that occurs immediately before the anchor in a counterclockwise traversal of the outer cycle is regarded as the first root of the Halin graph. We regard the interior vertex that is adjacent to the anchor as the root of the inner tree.

Proposition 3.1. The inner tree of a cubic Halin graph is a rooted binary tree.

Proof. We assign an ordering to the neighbors of the root by proceeding counterclockwise from the anchor vertex. At any vertex of the inner tree 


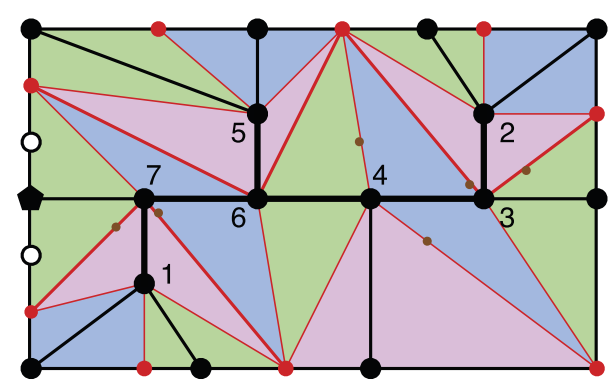

Figure 6: A Halin graph with quadrangulation and virtual roots.

with two children in the inner tree, the ordering of the children is induced by proceeding counterclockwise from the parent node.

A vertex with only one child in the inner tree has its third neighbor on the outer cycle. If the edge to this outer cycle vertex precedes the child in the inner tree, then that child is a right-child. Otherwise, it is a left-child. Of course, a vertex with no children in the inner tree is a leaf-node of the binary tree.

Theorem 3.2. The cubic Halin graphs are in bijective correspondence with the rooted binary trees.

Proof. An empty inner tree for a Halin graph corresponds to the notion of an empty binary tree, which lets us say that there is one binary tree with no vertices, corresponding to the Catalan number $c_{0}=1$. Our model for such a Halin graph is a circle with a diameter. One endpoint of the diameter is the anchor. Since the other endpoint is on the circle, the inner tree is empty.

The rooted binary tree corresponding to any given cubic Halin graph is the inner tree, as per Proposition 3.1. Conversely, let $T$ be a rooted binary tree. Draw a circle around it, without touching the tree $T$, place an anchor node on that circle, and draw an edge joining the root $r$ of the tree with the anchor node. If the root $r$ has only one child $v$ (in the inner tree), then draw an edge to a new node $w$ on the circle so that tree-node $v$ precedes cycle-node $w$ at root $r$ if $v$ is a left-child of $r$, and so that tree-node $v$ follows cycle-node $w$ if $v$ is a right-child.

We proceed breadth-first in tree $T$ as we join some of its vertices to new vertices on the circle. Thus, when we arrive at any particular vertex $u$, we have already fully installed its parent. The rules for drawing leaf-edges from vertex $u$ to new vertices on the circle depend on the number of children of vertex $u$, which can be 0,1 , or 2 . 
1. Install two consecutive vertices on the arc of the circle that are accessible to vertex $u$ without crossing any edges.

2. If $u$ is a left-child of its parent, then draw an edge from $u$ to a new vertex on the circle, so that the new edge follows the edge from $u$ to its child. Otherwise, draw an edge from $u$ to a new vertex on the circle so that the new edge precedes the edge to the child of $u$.

3. No new edges are drawn from vertex $u$.

It is clear that the resulting graph is a cubic Halin graph.

Corollary 3.3. The number of cubic Halin graphs with $n$ vertices in its inner tree is the Catalan number $c_{n}$.

We now give a recursive algorithm for calculating the pgd-vector of a cubic Halin graph that is the union of a tree $T$ with a cycle $C$, installed in the plane so that $C$ is the outer cycle. We are proceeding with a pre-order traversal of the binary tree (not just the inner tree).

1. Start at root vertex of the inner tree.

2. If the left-child is on the cycle $C$, then record the row-vector $A$ from Equation (4). Otherwise, inside a pair of parentheses, record the pgdvector of the cubic Halin graph for which that left-child is root of the inner tree.

3. Record the vp-matrix $M$ from Theorem 2.1 .

4. If the right-child is on the cycle $C$, then record the column vector $A^{t}$. Otherwise, after a left parenthesis, record the pgd-vector of the cubic Halin graph or which that right-child is root of the inner tree, followed by $)^{t}$, that is, a right parenthesis and then a transpose notation.

With pgd-vectors $A$ and $Y$ defined by Equations (4) and (5), the matrix formula for the pgd-vector of the Halin graph of Figure 6 is

$$
\left(A M A^{t}\right) M\left(\left(A M\left(A M\left(A M A^{t}\right)^{t}\right)^{t}\right) M\left(A M A^{t}\right)^{t}\right)^{t} .
$$

Replacing $\left(A M A^{t}\right)$ by $Y$ yields the simplified formula

$$
Y M\left(\left(A M\left(A M Y^{t}\right)^{t}\right) M Y^{t}\right)^{t}
$$

Either matrix formula yields the following genus polynomial:

$$
2+286 z+6912 z^{2}+31968 z^{3}+26368 z^{4} .
$$




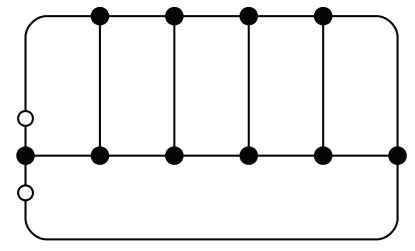

(a)

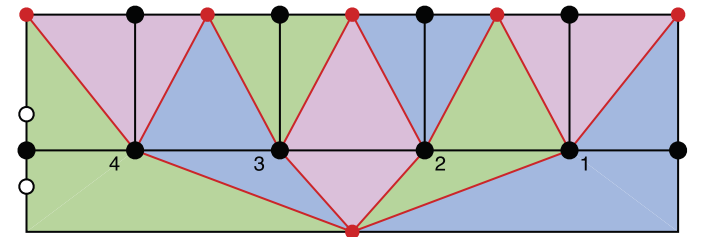

(b)

Figure 7: (a) A Halin graph isomorphic to the Ringel ladder $R L_{4}$.

(b) Quadrangulation of its plane imbedding.

\section{Log-concavity results for cubic Halin graphs}

We now consider some special sequences of cubic Halin graphs whose genus polynomials are provably log-concave. For this purpose, we rewrite the vpmatrix $M$ of Theorem 2.1 in the form

$$
\frac{1}{4}\left(\begin{array}{cccccc}
\frac{1}{2} v+2 z e_{2} & v & v & v & 4 e_{4} & 2 e_{3}+2 e_{4} \\
v & 4 e_{2} & 4 e_{3} & 4 e_{4} & 4 e_{5} & \frac{1}{z} v \\
2 e_{3}+2 z e_{5} & 4 e_{3} & 4 e_{3} & 4 e_{5} & 4 e_{5} & \frac{2}{z} e_{3}+2 e_{5} \\
v & 4 e_{4} & \frac{1}{z} v & 4 e_{4} & \frac{4}{z} e_{4} & \frac{1}{z} v \\
4 e_{3} & 4 e_{5} & \frac{4}{z} e_{3} & 4 e_{5} & \frac{4}{z} e_{5} & \frac{4}{z} e_{3} \\
2 e_{3}+2 e_{4} & \frac{2}{z} e_{1}+2 e_{6} & \frac{2}{z} e_{1}+2 e_{6} & \frac{2}{z} e_{4}+2 e_{5} & \frac{4}{z} e_{4} & \frac{1}{2 z^{2}} v+\frac{2}{z} e_{2}
\end{array}\right),
$$

where $v=(2,0,0,0,0,2 z)^{t}$, and where $e_{j}$ is the $j^{\text {th }}$ unit vector in $\mathbb{R}^{6}$.

\subsection{Revisiting Ringel ladders}

The cubic Halin graph in Figure 7(a) is recognizable as the Ringel ladder $R L_{4}$, whose genus polynomial is already known [7] to be log-concave.

From the quadrangulation in Figure 7(b), we see that the pgd-vectors of the corresponding infinite sequence of Halin graphs (i.e., the Ringel ladders) are of the form

$$
\left(\left(\cdots\left(A M A^{t}\right) \cdots M A^{t}\right) M A^{t}\right) M A^{t}
$$

where $A=(2,0,0,0,0,2 z)$, as in Equation (4).

\subsection{A second family of cubic Halin graphs}

We now seek an explicit general formula for the pdg-vectors of various infinite sequence of cubic Halin graphs exemplified by Figure 8(a). The general 


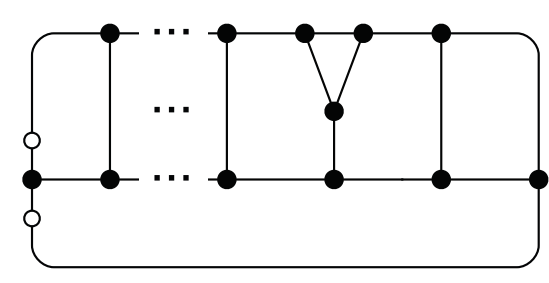

(a)

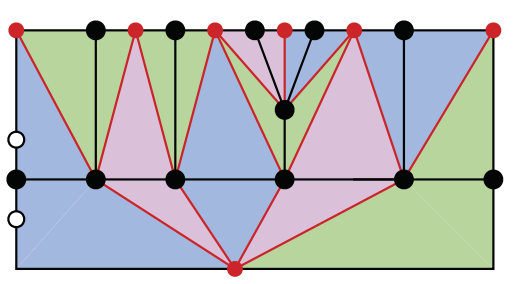

(b)

Figure 8: (a) A sequence of Halin graphs $Y I_{0}, Y I_{1}, Y I 2, \ldots$.

(b) Quadrangulation of a plane imbedding of $Y I_{2}$.

pattern of each of these infinite sequences is a rectangularized cycle with a subdivided horizontal diameter. From each interior vertex $v$ of this diameter, there is a tree, all of whose leaves (other than $v$ ) lie on the arc above the diameter. Each sequence is characterized by a fixed finite sequence of trees at the right, after which (proceeding leftward), all the remaining trees have only one edge.

In order to find such a formula, we define the following recurrence:

$$
\begin{aligned}
& F_{n}(z)=\left(F_{n-1}\right) M A^{t}, \\
& F_{1}(z)=\left(v_{1}, \ldots, v_{6}\right),
\end{aligned}
$$

where $A=(2,0,0,0,0,2 z)$. If we denote the $j^{\text {th }}$ coordinate of the vector $F_{n}(z)$ by $F_{n}^{(j)}$, we see from (9) that the coordinates $F_{n}^{(j)}$ satisfy the following recurrence system:

$$
\begin{aligned}
& F_{n}^{(1)}=\frac{1}{2} F_{n-1}^{(1)}+2 F_{n-1}^{(2)}+2 F_{n-1}^{(4)}+\frac{1}{2 z} F_{n-1}^{(6)}, \\
& F_{n}^{(2)}=z F_{n-1}^{(1)}+F_{n-1}^{(6)}, \\
& F_{n}^{(3)}=z F_{n-1}^{(1)}+2 F_{n-1}^{(3)}+4 F_{n-1}^{(5)}+F_{n-1}^{(6)}, \\
& F_{n}^{(4)}=z F_{n-1}^{(1)}+F_{n-1}^{(6)}, \\
& F_{n}^{(5)}=2 z F_{n-1}^{(3)}, \\
& F_{n}^{(6)}=\frac{z}{2} F_{n-1}^{(1)}+2 z F_{n-1}^{(2)}+2 z F_{n-1}^{(4)}+\frac{1}{2} F_{n-1}^{(6)}
\end{aligned}
$$

with the initial conditions (from $(10)) F_{1}^{(j)}=v_{j}$ for all $j=1,2, \ldots, 6$.

REMARK. The following theorem and corollary hold with $v_{1}, v_{2}, \ldots, v_{6}$ equal to arbitrary functions of $z$. 
Theorem 4.1. For $n \geq 2$, the vector $F_{n}(z)=\left(F_{n}^{(1)}, \ldots, F_{n}^{(6)}\right)$ is given by

$$
\begin{aligned}
& \left.F_{n}^{(1)}=v_{1} h_{n-1}+\frac{1}{2 z}\left(4 z v_{2}-z v_{1}+4 z v_{4}+v_{6}\right) h_{n-2}-4 z\left(v_{1} z-v_{6}\right)\right) h_{n-3}, \\
& F_{n}^{(2)}=v_{2} h_{n-1}+\left(z v_{1}-v_{2}+v_{6}\right) h_{n-2}-4 z\left(v_{2}-v_{4}\right) h_{n-3}, \\
& F_{n}^{(3)}=-\left(z v_{1}+v_{6}\right) h_{n-1}-\frac{1}{2}\left(v_{2}+v_{4}\right)\left(h_{n}-h_{n-1}\right) \\
& +\frac{1}{2 z}\left(z v_{2}+z v_{4}+v_{5}\right)\left(h_{n}^{\prime}-h_{n-1}^{\prime}\right)+\left(z v_{1}+v_{3}+v_{6}\right) h_{n-1}^{\prime}, \\
& F_{n}^{(4)}=v_{4} h_{n-1}+\left(z v_{1}-v_{4}+v_{6}\right) h_{n-2}+4 z\left(v_{2}-v_{4}\right) h_{n-3}, \\
& F_{n}^{(5)}=-\frac{1}{8}\left(2 z v_{1}-v_{2}-v_{4}+2 v_{6}\right)\left(h_{n}-h_{n-1}\right)-z\left(v_{2}+v_{4}\right) h_{n-1} \\
& +\frac{1}{2 z}\left(z^{2} v_{1}-z v_{2}+z v_{3}-z v_{4}-v_{5}+z v_{6}\right)\left(h_{n}^{\prime}-2 h_{n-1}^{\prime}\right) \\
& +2\left(z v_{2}+z v_{4}+v_{5}\right) h_{n-1}^{\prime} \text {, } \\
& F_{n}^{(6)}=v_{6} h_{n-1}+\frac{1}{2}\left(z v_{1}+4 z v_{2}+4 z v_{4}-v_{6}\right) h_{n-2}+4 z\left(z v_{1}-v_{6}\right) h_{n-3} \text {, }
\end{aligned}
$$

where $h_{n}=\sqrt{-8 z}^{n} U_{n}\left(\frac{1}{2 \sqrt{-8 z}}\right)$ and $h_{n}^{\prime}=\sqrt{-8 z}^{n} U_{n}\left(\frac{1}{\sqrt{-8 z}}\right)$.

Proof. We define the generating functions $F^{(j)}(t)=\sum_{n \geq 1} F_{n}^{(j)} t^{n}$, for $j=$ $1,2, \ldots, 6$. By multiplying the recurrence system above by $t^{n}$, next summing over $n \geq 2$, and then solving for $F^{(j)}(t)$, we obtain that

$$
\begin{aligned}
F^{(1)}(t) & =\frac{\left.2 z v_{1} t+\left(4 z v_{2}-z v_{1}+4 z v_{4}+v_{6}\right) t^{2}-8 z\left(v_{1} z-v_{6}\right)\right) t^{3}}{2 z\left(1-t-8 z t^{2}\right)}, \\
F^{(2)}(t) & =\frac{v_{2} t+\left(z v_{1}-v_{2}+v_{6}\right) t^{2}-4 z\left(v_{2}-v_{4}\right) t^{3}}{1-t-8 z t^{2}}, \\
F^{(3)}(t) & =\frac{v_{3} t+\left(z v_{1}-v_{3}+4 v_{5}+v_{6}\right) t^{2}}{\left(1-2 t-8 z t^{2}\right)\left(1-t-8 z t^{2}\right)} \\
& +\frac{4\left(z v_{2}-2 z v_{3}+z v_{4}-v_{5}\right) t^{3}-32 z v_{5} t^{4}}{\left(1-2 t-8 z t^{2}\right)\left(1-t-8 z t^{2}\right)}, \\
F^{(4)}(t) & =\frac{v_{4} t+\left(z v_{1}-v_{4}+v_{6}\right) t^{2}+4 z\left(v_{2}-v_{4}\right) t^{3}}{1-t-8 z t^{2}}, \\
F^{(5)}(t) & =\frac{v_{5} t+\left(2 z v_{3}-3 v_{5}\right) t^{2}+2\left(z^{2} v_{1}-z v_{3}+(1-4 z) v_{5}+z v_{6}\right) t^{3}}{\left(1-2 t-8 z t^{2}\right)\left(1-t-8 z t^{2}\right)} \\
& +\frac{8 z\left(z v_{2}-2 z v_{3}+z v_{4}+2 v_{5}\right) t^{4}}{\left(1-2 t-8 z t^{2}\right)\left(1-t-8 z t^{2}\right)},
\end{aligned}
$$




$$
F^{(6)}(t)=\frac{2 v_{6} t+\left(z v_{1}+4 z v_{2}+4 z v_{4}-v_{6}\right) t^{2}+8 z\left(z v_{1}-v_{6}\right) t^{3}}{2\left(1-t-8 z t^{2}\right)} .
$$

Using the fact that Chebyshev polynomials $U_{n}(x)$ of the second kind have the generating function $\frac{1}{1-2 x t+t^{2}}$, we obtain the conclusion.

Corollary 4.2. A closed formula for the generating function

$$
G(t)=\sum_{n \geq 1} g_{n} t^{n}=F^{(1)}(t)+\cdots+F^{(6)}(t)
$$

$i s$

$$
\begin{aligned}
G(t)=- & \frac{2 z\left(v_{1}+v_{3}\right)+(1+z)\left(v_{2}+v_{4}\right)+2\left(v_{5}+v_{6}\right)}{8 z}+\frac{(1-z)\left(z v_{1}-v_{6}\right) t}{2 z} \\
& +\frac{(1-z)\left(2\left(z v_{1}+v_{6}\right)(1+t)+\left(v_{2}+v_{4}\right)(1+8 z t-t)\right)}{8 z\left(1-t-8 z t^{2}\right)} \\
& +\frac{z\left(z v_{1}+v_{3}+v_{6}\right)(1+2 t)+\left(z v_{2}+z v_{4}+v_{5}\right)(1+4 z t-2 t)}{4 z\left(1-2 t-8 z t^{2}\right)} .
\end{aligned}
$$

Moreover, for $n \geq 2$, the polynomial $g_{n}$ is given by

$$
\begin{aligned}
g_{n} & =\frac{(1-z)\left(2\left(z v_{1}+v_{6}\right)\left(h_{n}+h_{n-1}\right)+\left(v_{2}+v_{4}\right)\left(h_{n}+(8 z-1) h_{n-1}\right)\right)}{8 z} \\
& +\frac{z\left(z v_{1}+v_{3}+v_{6}\right)\left(h_{n}^{\prime}+2 h_{n-1}^{\prime}\right)+\left(z v_{2}+z v_{4}+v_{5}\right)\left(h_{n}^{\prime}+2(2 z-1) h_{n-1}^{\prime}\right)}{4 z},
\end{aligned}
$$

where $h_{n}=\sqrt{-8 z}^{n} U_{n}\left(\frac{1}{2 \sqrt{-8 z}}\right)$ and $h_{n}^{\prime}=\sqrt{-8 z}^{n} U_{n}\left(\frac{1}{\sqrt{-8 z}}\right)$.

We are now ready to give applications of Corollary 4.2.

Example 4.3. Ringel ladders are illustrated by Figure \%. We apply Corollary 4.2 with $F_{1}(z)=\left(v_{1}, \ldots, v_{6}\right)=(2,0,0, \ldots, 2 z)=A$ (see (4)), which gives

$$
G(t)=-1+\frac{(1-z)(1+t)}{1-t-8 z t^{2}}+\frac{z(1+2 t)}{1-2 t-8 z t^{2}}
$$

and for $n \geq 1$,

$$
g_{n}=(1-z)\left(h_{n}+h_{n-1}\right)+z\left(h_{n}^{\prime}+2 h_{n-1}^{\prime}\right) .
$$

Note that

$$
\sqrt{-8 z}^{n} U_{n}\left(\frac{1}{m \sqrt{-8 z}}\right)=\sum_{j \geq 0}\left(\begin{array}{c}
n-j \\
j
\end{array}\right)(2 / m)^{n-2 j} 8^{j} z^{j} .
$$


Thus, the coefficient of $z^{j}$ in $g_{n}$ is given by

$$
\begin{aligned}
g_{n}(j) & =\left[\left(\begin{array}{c}
n-j \\
j
\end{array}\right)+\left(\begin{array}{c}
n-1-j \\
j
\end{array}\right)\right] 8^{j} \\
& +\left[\left(\begin{array}{c}
n+1-j \\
j-1
\end{array}\right)+\left(\begin{array}{c}
n-j \\
j-1
\end{array}\right)\right]\left(2^{n-1+j}-8^{j-1}\right) .
\end{aligned}
$$

This formula is consistent with Theorem 5.2 of [7].

Example 4.4. For the sequence of cubic Halin graphs $Y I_{n}$ of Figure 8, we apply Corollary 4.2 with

$$
\begin{aligned}
F_{1}(z) & =\left(v_{1}, \ldots, v_{6}\right)=\left(A M A^{t}\right) M\left(A M A^{t}\right)^{t}=Y M Y^{t} \text { as in }(5) \\
& =2\left((1+20 z), 2 z(1+4 z), 6 z(1+4 z), 6 z(1+4 z), 16 z^{2}, z(1+20 z)\right),
\end{aligned}
$$

which gives

$$
\begin{aligned}
G(t)= & -3-41 z-20 z^{2} \\
+ & \frac{(1-z)(1+20 z)(1+t)+2(1-z)(1+4 z)(1+8 z t-t)}{1-t-8 z t^{2}} \\
& +\frac{4 z(1+8 z)(1+2 t)+4 z(3+4 z)(1+4 z t-2 t)}{1-2 t-8 z t^{2}} .
\end{aligned}
$$

Moreover, for $n \geq 2$, the polynomial $g_{n}$ is given by

$$
\begin{aligned}
g_{n} & =(1-z)(3+28 z) h_{n}+(1-z)\left(64 z^{2}+28 z-1\right) h_{n-1} \\
& +16 z(1+3 z) h_{n}^{\prime}+16 z\left(4 z^{2}+5 z-1\right) h_{n-1}^{\prime} .
\end{aligned}
$$

Thus, by (11) we establish that the coefficient of $z^{j}$ in $g_{n}$ is given by

$$
\begin{aligned}
\frac{g_{n}(j)}{4 \cdot 8^{j-2}} & =48\left(\begin{array}{c}
n-j \\
j
\end{array}\right)-16\left(\begin{array}{c}
n-1-j \\
j
\end{array}\right)+\left(\begin{array}{c}
n+1-j \\
j-1
\end{array}\right)\left(50+2^{n+7-2 j}\right) \\
& +\left(\begin{array}{c}
n-j \\
j-1
\end{array}\right)\left(58-2^{n+6-2 j}\right)+\left(\begin{array}{c}
n+2-j \\
j-2
\end{array}\right)\left(3 \cdot 2^{n+6-2 j}-7\right) \\
& +\left(\begin{array}{c}
n+1-j \\
j-2
\end{array}\right)\left(9+5 \cdot 2^{n+5-2 j}\right)+2\left(\begin{array}{c}
n+2-j \\
j-3
\end{array}\right)\left(2^{n+11-2 j}-1\right) .
\end{aligned}
$$




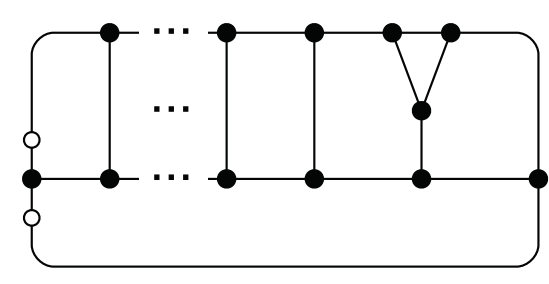

(a)

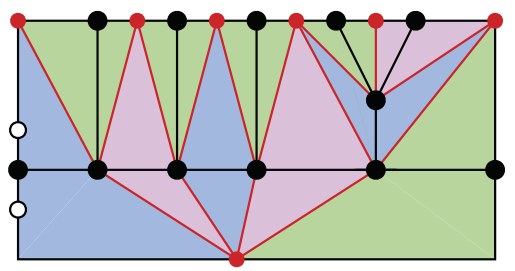

(b)

Figure 9: (a) A sequence of Halin graphs $H Y_{0}, H Y_{1}, H Y_{2}, \ldots$

(b) Quadrangulation of a plane imbedding of $\mathrm{HY}_{2}$.

Example 4.5. The sequence of cubic Halin graphs depicted in Figure 9 has

$$
\begin{aligned}
F_{1}(z) & =\left(v_{1}, \ldots, v_{6}\right)=A M\left(A M A^{t}\right)^{t}=A M Y^{t} \\
& =2\left((1+8 z), 2 z, 2 z, 6 z, 4 z^{2}, z(1+8 z)\right),
\end{aligned}
$$

Applying Corollary 4.2 gives

$$
\begin{aligned}
G(t) & =-3-13 z+\frac{(1-z)(3+8 z)-(1-z)(1-24 z) t}{1-t-8 z t^{2}} \\
& +\frac{8 z(1+z+(5 z-1) t)}{1-2 t-8 z t^{2}} .
\end{aligned}
$$

Moreover, for $n \geq 2$, the polynomial $g_{n}$ is given by

$g_{n}=(1-z)\left((3+8 z) h_{n}-(1-24 z) h_{n-1}\right)+8 z\left((1+z) h_{n}^{\prime}+(5 z-1) h_{n-1}^{\prime}\right)$.

Thus, by (11) we establish that the coefficient of $z^{j}$ in $g_{n}$ is given by

$$
\begin{aligned}
\frac{g_{n}(j)}{8^{j-1}} & =24\left(\begin{array}{c}
n-j \\
j
\end{array}\right)-8\left(\begin{array}{c}
n-1-j \\
j
\end{array}\right)+\left(\begin{array}{c}
n-j+1 \\
j-1
\end{array}\right)\left(2^{n-2 j+5}+5\right) \\
& +\left(\begin{array}{c}
n-j+2 \\
j-2
\end{array}\right)\left(2^{n-2 j+4}-1\right)+\left(\begin{array}{c}
n-j+1 \\
j-2
\end{array}\right)\left(5 \cdot 2^{n-2 j+3}-3\right) \\
& -\left(\begin{array}{c}
n-j \\
j-1
\end{array}\right)\left(2^{n-2 j+4}-25\right) .
\end{aligned}
$$

Example 4.6. For the following vectors $F_{1}(z)$, by using similar techniques as in above examples, one can get explicit formulas for the genus polynomials, and prove that they are log-concave polynomials: 
(a) $F_{1}=A M\left(A M Y^{t}\right)^{t}$,

(b) $F_{1}=A M\left(A M\left(A M Y^{t}\right)^{t}\right)^{t}$,

(c) $F_{1}=Y M\left(Y M Y^{t}\right)^{t}$.

\section{Log-concavity}

In this final section, we provide a procedure via Theorem 5.1 to prove that the genus polynomials of our example sequences and similar sequences of cubic Halin graphs are log-concave. Note that most of the steps of Theorem 5.1 require the help of a mathematical programming system (such as Maple or Mathematica).

Theorem 5.1. Let $n \geq n_{0}$ and let $j=j_{0}, j_{0}+1, \ldots, \frac{n-m}{2}$, where $n_{0}$ and $m$ are any constants with $n_{0} \geq m$. Assume that the function $p_{n}(j)$ can be written as a sum

$$
p_{n}(j)=\sum_{\ell=0}^{r} a_{n}^{(\ell)}(j) t^{\ell(n-s j)}
$$

where $a_{n}^{(\ell)}(j)$ is a polynomial in $n$ and $j$, for $\ell=0,1, \ldots, r$, such that

(i) There exist constants $m^{\prime}>0$ and $q>0$ with $m^{\prime}<m$ and $t^{n-s j} \geq$ $q(n-s j)$, for all $j=j_{0}, j_{0}+1, \ldots, \frac{n-m}{2}$ and $n>n_{0}$;

(ii) For all $\ell=r, r-1, \ldots, 1$, for all $j=j_{0}, j_{0}+1, \ldots, \frac{n-m}{2}$, and for $n>n_{0}$, the polynomial

$$
q_{n}^{(\ell)}(j)=\sum_{d=\ell}^{r} a_{n}^{(d)}(j)(q(n-s j))^{\ell-d+1}
$$

is non-negative;

(iii) For all $j=j_{0}, j_{0}+1, \ldots, \frac{n-m}{2}$ and for $n>n_{0}$, the polynomial

$$
q_{n}^{(0)}(j)=\sum_{d=0}^{r} a_{n}^{(d)}(j)(q(n-s j))^{\ell}
$$

is non-negative.

Then $p_{n}(j) \geq 0$, for all $j=j_{0}, j_{0}+1, \ldots, \frac{n-m}{2}$ and $n>n_{0}$. 
Proof. Let $j_{0}, j_{0}+1, \ldots, \frac{n-m}{2}$ and $n>n_{0}$. Then, by (i) and (ii) we have

$$
\begin{aligned}
p_{n}(j) & =\sum_{\ell=0}^{r} a_{n}^{(\ell)}(j) t^{\ell(n-s j)} \geq \sum_{\ell=0}^{r-1} a_{n}^{(\ell)}(j) t^{\ell(n-s j)}+t^{(r-1)(n-s j)} q_{n}^{(r)}(j) \\
\geq & \sum_{\ell=0}^{r-2} a_{n}^{(\ell)}(j) t^{\ell(n-s j)}+t^{(r-2)(n-s j)} q_{n}^{(r-1)}(j) \\
& \vdots \\
& \geq a_{n}^{(0)}(j)+t^{(r-r)(n-s j)} q_{n}^{(1)}(j)=q_{n}^{(0)} \geq 0
\end{aligned}
$$

which completes the proof.

In what follows, we use this theorem to prove the log-concavity of the sequence of Halin graphs corresponding to Figure 8.

Theorem 5.2. The genus polynomial of any graph $Y I_{n}$, as defined in Example 4.4, is log-concave.

Proof. Let $g_{n}(j)$ be defined as in (13). By direct calculations, with the help of a mathematical programming sytem, we can see that the function $g_{n}(j)$ can be expressed as

$$
\begin{aligned}
g_{n}^{\prime}(j) & =\left(g_{n}(j)\right)^{2}-g_{n}(j-1) g_{n}(j+1) \\
& =\frac{8^{2 j-2} p_{n}(j)(n-2-j) !(n-1-j) !}{4 j !(j+1) !(n+5-2 j) !(n+7-2 j) !} \\
& =\frac{8^{2 j-2}\left(a_{n}(j)+b_{n}(j) 2^{n-2 j}+c_{n}(j) 2^{2 n-4 j}\right)(n-2-j) !(n-1-j) !}{4 j !(j+1) !(n+5-2 j) !(n+7-2 j) !},
\end{aligned}
$$

for all $j=0,1,2, \ldots, \frac{n+5}{2}$. Fix $n_{0}=6$. (It is not hard to see that $g_{n}(z)$ is a log-concave polynomial, for $n=0,1, \ldots, n_{0}-1$.) Note that $p_{n}\left(\frac{n+5}{2}\right)=0$,

$$
\begin{aligned}
& p_{n}\left(\frac{n+4}{2}\right)=\frac{3}{2048} n^{2}\left(n^{2}-36\right)\left(n^{2}-16\right)^{2}\left(n^{2}-4\right)^{2}(n+5)^{2}, \\
& p_{n}\left(\frac{n+3}{2}\right)=\frac{3}{1024}\left(n^{2}-25\right)\left(n^{2}-9\right)^{2}\left(n^{2}-1\right)^{2}\left(3 n^{2}+34 n+147\right)^{2},
\end{aligned}
$$




$$
\begin{aligned}
& p_{n}\left(\frac{n+2}{2}\right)=\frac{1}{2048} n^{2}\left(n^{2}-16\right)\left(n^{2}-4\right)^{2}\left(397 n^{6}+13980 n^{5}+220735 n^{4}\right. \\
& \left.+1993620 n^{3}+10632868 n^{2}+30850320 n+37347840\right), \\
& p_{n}\left(\frac{n+1}{2}\right)=\frac{3}{2048}\left(n^{2}-9\right)\left(n^{2}-1\right)^{2}\left(747 n^{8}+35544 n^{7}+746372 n^{6}\right. \\
& +9088392 n^{5}+69658658 n^{4}+333822696 n^{3}+939437828 n^{2} \\
& +1222667448 n+765895275) \text {, } \\
& p_{n}\left(\frac{n}{2}\right)=\frac{1}{2048} n^{2}\left(n^{2}-4\right)\left(11291 n^{10}+675250 n^{9}+17648355 n^{8}\right. \\
& +264445080 n^{7}+2482032168 n^{6}+14576852640 n^{5} \\
& +52682726320 n^{4}+116037096320 n^{3}+142037711616 n^{2} \\
& +69500252160 n+178362777600) \text {, } \\
& p_{n}\left(\frac{n-1}{2}\right)=\frac{3}{2048}\left(n^{2}-1\right)\left(17919 n^{12}+1288812 n^{11}+40367354 n^{10}\right. \\
& +716390340 n^{9}+7773709197 n^{8}+49651550136 n^{7} \\
& +170799321372 n^{6}+389074688520 n^{5}+1094324663209 n^{4} \\
& \text { - } 2218709032548 n^{3}-1350300915126 n^{2}+3681801409140 n \\
& +3248256660075) \text {, } \\
& p_{n}(0)=1024(n-1)(n+7)(n+6) \prod_{j=0}^{5}(n+j)^{2} \text {, }
\end{aligned}
$$

which shows that $g_{n}^{\prime}(j) \geq 0$, for all $j=0, \frac{n-1}{2}, \frac{n}{2}, \ldots, \frac{n+5}{2}$, where $n \geq n_{0}$. Thus, it remains to show that $p_{n}(j) \geq 0$ for all $n \geq n_{0}$ and $j=j_{0}, j_{0}+$ $1, \ldots, \frac{n-m}{2}$, where $j_{0}=1$ and $m=2$. With the help of a mathematical programming system, we have

$$
\begin{aligned}
& \frac{1024 c_{n}(j)}{j(j+1)} \\
& =8 j^{2}(j+4)(j-1)\left(11 j^{8}+286 j^{7}+3071 j^{6}+17416 j^{5}+53039 j^{4}+54334 j^{3}\right. \\
& \left.-57201 j^{2}+8604 j-19080\right)+4 j\left(4 j^{11}+402 j^{10}+8939 j^{9}+90957 j^{8}\right. \\
& +497247 j^{7}+1395669 j^{6}+1177232 j^{5}-1971312 j^{4}-1395206 j^{3}+390324 j^{2} \\
& +181584 j+228960)(n-2 j)+2\left(104 j^{11}+6136 j^{10}+109656 j^{9}+926037 j^{8}\right. \\
& +4059491 j^{7}+8116030 j^{6}+2237454 j^{5}-8016639 j^{4}-3138925 j^{3}+284896 j^{2}
\end{aligned}
$$




$$
\begin{aligned}
& +1161360 j+152640)(n-2 j)^{2}+\left(1236 j^{10}+53364 j^{9}+776436 j^{8}\right. \\
& +5320878 j^{7}+17795955 j^{6}+23122341 j^{5}-2142995 j^{4}-16365059 j^{3} \\
& \left.-5818844 j^{2}+1555440 j+863136\right)(n-2 j)^{3}+2\left(2208 j^{9}+74391 j^{8}\right. \\
& +874547 j^{7}+4695037 j^{6}+11291981 j^{5}+8632927 j^{4}-3562392 j^{3} \\
& \left.-5591366 j^{2}-1190365 j+331328\right)(n-2 j)^{4}+\left(10488 j^{8}+280134 j^{7}\right. \\
& +2600136 j^{6}+10440012 j^{5}+16923714 j^{4}+6073626 j^{3}-6496343 j^{2} \\
& -4606231 j-301592)(n-2 j)^{5}+2\left(8642 j^{7}+181381 j^{6}+1281383 j^{5}\right. \\
& \left.+3639157 j^{4}+3627281 j^{3}-28577 j^{2}-1482453 j-394844\right)(n-2 j)^{6} \\
& +\left(20037 j^{6}+321783 j^{5}+1647039 j^{4}+3075621 j^{3}+1513563 j^{2}-771963 j\right. \\
& -534076)(n-2 j)^{7}+6\left(2706 j^{5}+31869 j^{4}+110650 j^{3}+120008 j^{2}\right. \\
& +5457 j-29684)(n-2 j)^{8}+\left(8978 j^{4}+72980 j^{3}+155567 j^{2}+71019 j\right. \\
& -28092)(n-2 j)^{9}+2\left(1622 j^{3}+8319 j^{2}+8965 j-116\right)(n-2 j)^{10} \\
& +\left(713 j^{2}+1975 j+620\right)(n-2 j)^{11}+4(21 j+22)(n-2 j)^{12}+4(n-2 j)^{13} .
\end{aligned}
$$

Note that each coefficient $(n-2 j)^{k}$ in $c_{n}(j)$ is non-negative (to see this, we expand it as a Taylor series at $j=1$ ) for all $j=j_{0}, j_{0}+1, \ldots, \frac{n-m}{2}$ and $n \geq n_{0}$, which implies that $c_{n}(j) \geq 0$. By considering the polynomial $b_{n}^{\prime}(j)=b_{n}(j)+2 c_{n}(j)(n-2 j)$, we can write

$$
\begin{aligned}
& \frac{b_{n}^{\prime}(j)}{8 j} \\
& =8 j(j+1)^{2}\left(3359 j^{10}+137587 j^{9}+2474130 j^{8}+25534710 j^{7}+165097407 j^{6}\right. \\
& +672129051 j^{5}+1671707200 j^{4}+2557644380 j^{3}+2543193744 j^{2} \\
& -165807648 j-1143486720)+4(j+1)\left(7747 j^{12}+412775 j^{11}+9333398 j^{10}\right. \\
& +118822058 j^{9}+942021519 j^{8}+4771988847 j^{7}+15308677484 j^{6} \\
& +31451733376 j^{5}+44615065324 j^{4}+34671470368 j^{3}-173351232 j^{2} \\
& -13052970144 j-2472768000)(n-1-2 j)+\left(108612358968 j^{7}\right. \\
& -45138303360+26012075984 j^{8}+3968242096 j^{9}+292813370748 j^{6} \\
& +4132 j^{13}+9325299320 j^{2}+449831171016 j^{3}+673112160978 j^{4}
\end{aligned}
$$




$$
\begin{aligned}
& +538602971756 j^{5}-146796524832 j+580702 j^{12}+379199116 j^{10} \\
& \left.+21236192 j^{11}\right)(n-1-2 j)^{2}+\left(80626832420 j^{7}-88533656352\right. \\
& +15572317741 j^{8}+1842574904 j^{9}+260687588355 j^{6}+187233730744 j^{2} \\
& +699548570060 j^{3}+798915799817 j^{4}+551850290224 j^{5}-166611425240 j \\
& \left.+53734 j^{12}+127490377 j^{10}+4498816 j^{11}\right)(n-1-2 j)^{3} \\
& +\left(35675350102 j^{7}-98278308272+5294994995 j^{8}+451108199 j^{9}\right. \\
& +143683488548 j^{6}+306497318284 j^{2}+648436841636 j^{3}+603760615611 j^{4} \\
& \left.+361133337659 j^{5}-90643987752 j+19764754 j^{10}+319116 j^{11}\right)(n-1-2 j)^{4} \\
& +\left(9625397822 j^{7}-68165947912+1025337775 j^{8}+55508120 j^{9}\right. \\
& +50546339680 j^{6}+263400467899 j^{2}+394239255688 j^{3}+307651107681 j^{4} \\
& \left.+156646603268 j^{5}-4752345186 j+1137885 j^{10}\right)(n-1-2 j)^{5} \\
& +\left(1549116448 j^{7}-30446407420+105024767 j^{8}+2691309 j^{9}\right. \\
& +11334565970 j^{6}+143251611327 j^{2}+164418909245 j^{3}+108006666476 j^{4} \\
& \left.+45392365846 j^{5}+26966512544 j\right)(n-1-2 j)^{6}+\left(136322798 j^{7}\right. \\
& -8455327772+4401646 j^{8}+1564079386 j^{6}+52546612872 j^{2}+47842040960 j^{3} \\
& \left.+26102159596 j^{4}+8659627328 j^{5}+20007371186 j\right)(n-1-2 j)^{7} \\
& +\left(5035206 j^{7}-1160961432+120783064 j^{6}+13283885674 j^{2}\right. \\
& \left.+9683554956 j^{3}+4252205974 j^{4}+1041006618 j^{5}+7810138580 j\right)(n-1-2 j)^{8} \\
& +\left(78816648+3986757 j^{6}+2306804195 j^{2}+1332617530 j^{3}+444672304 j^{4}\right. \\
& \left.+71304732 j^{5}+1929407114 j\right)(n-1-2 j)^{9}+\left(69916004+267949619 j^{2}\right. \\
& \left.+118478031 j^{3}+26882473 j^{4}+2117297 j^{5}+312314304 j\right)(n-1-2 j)^{10} \\
& +2\left(356273 j^{4}+3055873 j^{3}+9809533 j^{2}+16224399 j+7329122\right)(n-1-2 j)^{11} \\
& +4\left(34571 j^{3}+199944 j^{2}+501349 j+407656\right)(n-1-2 j)^{12} \\
& +8\left(1653 j^{2}+7604 j+12223\right)(n-1-2 j)^{13}+64(7 j+39)(n-1-2 j)^{14} \text {. }
\end{aligned}
$$


Note that each coefficient $(n-1-2 j)^{k}$ in $b_{n}^{\prime}(j)$ is non-negative (to see this, we need only expand it as a Taylor series at $j=1$ ) for all $j=$ $j_{0}, j_{0}+1, \ldots, \frac{n-m}{2}$ and $n \geq n_{0}$, which implies that $b_{n}^{\prime}(j) \geq 0$. By considering the polynomial

$$
b_{n}^{\prime \prime}(j)=a_{n}(j)+2 b_{n}(j)(n-2 j)+4 c_{n}(j)(n-2 j)^{2},
$$

we can write

$$
\begin{aligned}
& \frac{b_{n}^{\prime \prime}(j)}{8 j} \\
& =84(2+j)(j+1)\left(738 j^{11}+39107 j^{10}+917633 j^{9}+12468630 j^{8}\right. \\
& +107337732 j^{7}+594633627 j^{6}+2072871045 j^{5}+4501421380 j^{4} \\
& \left.+6663188260 j^{3}+5522412936 j^{2}+606323952 j-2082767040\right)+\left(39252 j^{13}\right. \\
& +3004694 j^{12}+96229988 j^{11}+1738239628 j^{10}+19805704462 j^{9} \\
& +148564775634 j^{8}+740545509960 j^{7}+2462154899440 j^{6} \\
& +5606752930834 j^{5}+8882467342316 j^{4}+9113367582272 j^{3} \\
& \left.+4427711474352 j^{2}-856666567008 j-1349274426624\right)(n-2-2 j) \\
& +\left(4132 j^{13}+741904 j^{12}+36647336 j^{11}+891637621 j^{10}\right. \\
& +12798066837 j^{9}+116420182998 j^{8}+687039446306 j^{7} \\
& +2648830069601 j^{6}+6821967996661 j^{5}+12073498551092 j^{4} \\
& +14177247540744 j^{3}+8764452468528 j^{2}-109247190144 j \\
& -2241928217376)(n-2-2 j)^{2}+\left(53734 j^{12}+5775280 j^{11}+217928243 j^{10}\right. \\
& +4255915080 j^{9}+49260228421 j^{8}+355617809474 j^{7}+1629417776429 j^{6} \\
& +4838323990676 j^{5}+9642997729381 j^{4}+12868013286330 j^{3} \\
& \left.+9724631343008 j^{2}+1305605888632 j-2115368087952\right)(n-2-2 j)^{3} \\
& +\left(319116 j^{11}+25454179 j^{10}+769018434 j^{9}+12151112985 j^{8}\right. \\
& +112162848282 j^{7}+630133600870 j^{6}+2210638290031 j^{5}+5035255325010 j^{4} \\
& +7633112561321 j^{3}+6895083660004 j^{2}+1857495684480 j \\
& -1242677879448)(n-2-2 j)^{4}+\left(1137885 j^{10}+71655974 j^{9}+1747920943 j^{8}\right.
\end{aligned}
$$




$$
\begin{aligned}
& +22064846804 j^{7}+158665585572 j^{6}+678667297916 j^{5}+1805092282349 j^{4} \\
& +3128414069026 j^{3}+3338181686847 j^{2}+1353036592424 j \\
& -457675977244)(n-2-2 j)^{5}+\left(2691309 j^{9}+135836289 j^{8}+2644361802 j^{7}\right. \\
& +25999935052 j^{6}+141592172304 j^{5}+453110540038 j^{4}+910224394831 j^{3} \\
& \left.+1142893366573 j^{2}+630308256138 j-92383564512\right)(n-2-2 j)^{6} \\
& +2\left(2200823 j^{8}+88302223 j^{7}+1336933575 j^{6}+9904363132 j^{5}\right. \\
& +39794523636 j^{4}+94814236258 j^{3}+140573505160 j^{2}+100913577315 j \\
& -105330538)(n-2-2 j)^{7}+\left(5035206 j^{7}+156663877 j^{6}+1778027571 j^{5}\right. \\
& +9581538085 j^{4}+28085512791 j^{3}+49752910582 j^{2}+45655305104 j \\
& +6053721216)(n-2-2 j)^{8}+\left(3986757 j^{6}+92477702 j^{5}+752687064 j^{4}\right. \\
& \left.+2883966350 j^{3}+6250754895 j^{2}+7322812940 j+2017829940\right)(n-2-2 j)^{9} \\
& +\left(2117297 j^{5}+34720479 j^{4}+194833981 j^{3}+540326625 j^{2}+819453618 j\right. \\
& +369242592)(n-2-2 j)^{10}+2\left(356273 j^{4}+3885577 j^{3}+15123925 j^{2}\right. \\
& +30710759 j+21380714)(n-2-2 j)^{11}+4\left(34571 j^{3}+242922 j^{2}+709245 j\right. \\
& +782238)(n-2-2 j)^{12}+8\left(1653 j^{2}+8388 j+16591\right)(n-2-2 j)^{13} \\
& +64(7 j+39)(n-2-2 j)^{14} .
\end{aligned}
$$

Note that each coefficient $(n-2-2 j)^{k}$ in $b_{n}^{\prime \prime}(j)$ is non-negative. To see this, we expand it as a Taylor series at $j=1$, for all $j=j_{0}, j_{0}+1, \ldots, \frac{n-m}{2}$ and $n \geq n_{0}$, which implies that $b_{n}^{\prime \prime}(j) \geq 0$. Therefore, by Theorem 5.1 , the polynomial $g_{n}^{\prime}(j) \geq 0$ for all $j=j_{0}, j_{0}+1, \ldots, \frac{n-m}{2}$ and $n \geq 6$. It follows that the polynomial $g_{n}(z)$ is log-concave.

Theorem 5.3. The genus polynomials of the cubic Halin graphs defined in Examples 4.5 and 4.6 are log-concave.

Proof. Proof that the cubic Halin graph sequence of Example 4.5 has logconcave genus polynomials is given in Appendix A. Proofs of log-concavity for Example 4.6 are similar. 


\section{Conclusions and conjectures}

We have seen here how the use of vectorized production matrices, which were introduced in [6] for calculating genus distributions of graphs that are ring-like in structure, enables us to give formulas and to prove log-concavity for genus distributions of various infinite sequences of cubic Halin graphs. In the course of this, we have established a bijective correspondence between rooted cubic Halin graphs and rooted binary trees.

Like iterated claws [5], Ringel ladders [7], and bar-rings [6] of copies of $K_{4}$, all of which have previously been proved to have log-concave genus distributions, Halin graphs have treewidth 3. However, whereas these previously investigated families of graphs are linear or ring-like in structure, Halin graphs are tree-like in structure, which is a fully general kind of structure.

We conclude with two conjectures regarding the log-concavity of the genus distributions of several infinite sequences of cubic Halin graphs. Of course, both conjectures are true if the general conjecture that all graphs have log-concave genus polynomials is true.

Conjecture 6.1. Consider the sequence of cubic Halin graphs with genus polynomial formulas given by the recursion (9) with initial condition (10). Extend Examples 4.3-4.6 by showing that the genus polynomial $F_{n}(z)$ is log-concave for any initial pgd-vector $F_{1}(z)$.

It is not hard to extend Theorem 4.1 and its proof for other sequences of cubic Halin graphs. Here, let us give one more example. We define the following recurrence:

$$
\begin{aligned}
& P_{n}(z)=\left(P_{n-1}\right) M Y^{t}, \\
& P_{1}(z)=\left(v_{1}, \ldots, v_{6}\right) .
\end{aligned}
$$

If we denote the $j^{\text {th }}$ coordinate of the vector $P_{n}(z)$ by $P_{n}^{(j)}$, we see from $(15)$ that the coordinates $P_{n}^{(j)}$ satisfy the following recurrence system:

$$
\begin{aligned}
& P_{n}^{(1)}=\frac{1+8 z}{2} P_{n-1}^{(1)}+2 P_{n-1}^{(2)}+4 P_{n-1}^{(4)}+\frac{1+8 z}{2 z} P_{n-1}^{(6)}, \\
& P_{n}^{(2)}=z P_{n-1}^{(1)}+4 z P_{n-1}^{(2)}+P_{n-1}^{(6)}, \\
& P_{n}^{(3)}=z P_{n-1}^{(1)}+4 z P_{n-1}^{(2)}+2(1+4 z) P_{n-1}^{(3)}+8 P_{n-1}^{(5)}+P_{n-1}^{(6)}, \\
& P_{n}^{(4)}=3 z P_{n-1}^{(1)}+4 z P_{n-1}^{(2)}+8 z P_{n-1}^{(4)}+3 P_{n-1}^{(6)},
\end{aligned}
$$




$$
\begin{aligned}
& P_{n}^{(5)}=2 z^{2} P_{n-1}^{(3)}+6 z P_{n-1}^{(3)}+8 z P_{n-1}^{(5)}+2 z P_{n-1}^{(6)} \\
& P_{n}^{(6)}=\frac{z(1+8 z)}{2} P_{n-1}^{(1)}+2 z P_{n-1}^{(2)}+4 z P_{n-1}^{(4)}+\frac{1+8 z}{2} P_{n-1}^{(6)}
\end{aligned}
$$

with the initial conditions (from (16)) $P_{1}^{(j)}=v_{j}$ for all $j=1,2, \ldots, 6$. Again, note that the above recurrences system holds with $v_{1}, v_{2}, \ldots, v_{6}$ equal to arbitrary functions of $z$. By similar arguments as in the proof of Theorem 4.1 , we establish that the generating function $G(t)=\sum_{n \geq 1}\left(\sum_{j=1}^{6} P_{n}^{(j)}\right) t^{n}$ is given by

$$
Q(t)=\frac{Q^{\prime}(t) t}{Q^{\prime \prime}(t)},
$$

where

$$
\begin{aligned}
Q^{\prime \prime}(t) & =2 z\left(1-(1+20 z) t-16 z(1-8 z) t^{2}+64 z^{2}(1-4 z) t^{3}\right) \\
& \cdot\left(1-2(1+8 z) t-32 z(1-2 z) t^{2}\right) \\
Q^{\prime}(t) & =2 z \sum_{i=1}^{6} v_{i} \\
& -\left(z\left(5+53 z+12 z^{2}\right) v_{1}+2 z(1+22 z)\left(v_{2}+v_{3}\right)-2 z(1-24 z) v_{4}\right. \\
& \left.-2 z(5-28 z) v_{5}-\left(1+13 z-60 z^{2}\right) v_{6}\right) t+2\left(z \left(1-9 z+314 z^{2}\right.\right. \\
& \left.-152 z^{3}\right) v_{1}-2 z\left(1+18 z-96 z^{2}\right) v_{2}-14 z^{2}(1-12 z) v_{3} \\
& -2 z\left(3+32 z-112 z^{2}\right) v_{4}-2 z\left(3+64 z-144 z^{2}\right) v_{5} \\
& \left.-\left(1+15 z+190 z^{2}-360 z^{3}\right) v_{6}\right) t^{2}+32 z\left(2 z\left(1+11 z-69 z^{2}+44 z^{3}\right) v_{1}\right. \\
& -z\left(1-27 z+52 z^{2}\right) v_{2}+2 z^{2}(2-16 z) v_{3}-z\left(5-39 z+60 z^{2}\right) v_{4} \\
& \left.-2 z\left(3-30 z+40 z^{2}\right) v_{5}-2\left(1+7 z-63 z^{2}+68 z^{3}\right) v_{6}\right) t^{3} \\
& -128 z^{2}\left(z\left(-3+47 z-138 z^{2}+88 z^{3}\right) v_{1}-2 z(1-4 z)(2-3 z)\left(v_{2}+v_{4}\right)\right. \\
& +2 z^{2}(1-4 z) v_{3}-2 z(1-4 z)(3-4 z) v_{5} \\
& \left.+\left(3-47 z+142 z^{2}-104 z^{3}\right) v_{6}\right) t^{4} \\
& -2048 z^{3}(1-z)(1-2 z)(1-4 z)\left(v_{1} z-v_{6}\right) t^{5} . \\
&
\end{aligned}
$$




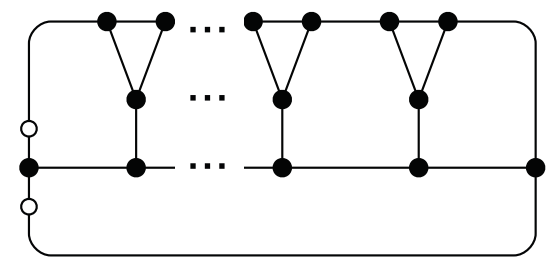

(a)

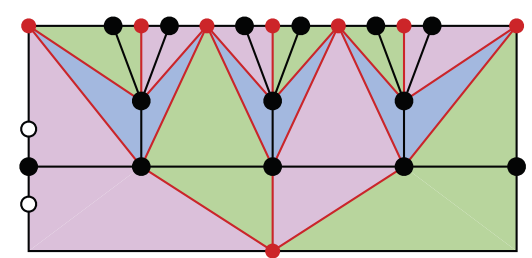

(b)

Figure 10: (a) A sequence of Halin graphs $A Y_{0}, A Y_{1}, A Y_{2}, \ldots$.

(b) Quadrangulation of a plane imbedding of $A Y_{3}$.

Conjecture 6.2. We define the polynomial $q_{n}(z)$ to be the coefficient of $t^{n}$ in the generating function

$$
Q(t)=\sum_{n \geq 1} q_{n}(z) t^{n},
$$

for $Q(t)$ as in (17). Then the genus polynomials for the sequence of cubic Halin graphs with genus polynomial $q_{n}(z)$ are log-concave for any initial vector $P_{1}(z)=\left(v_{1}, v_{2}, \ldots, v_{6}\right)$.

For example, if $P_{1}(z)=\left(v_{1}, v_{2}, \ldots, v_{6}\right)=A=(2,0,0,0,0,2 z)$, then $Q(t)$ is the generating function for the genus polynomial

$$
\left(\left(\left(A M Y^{t}\right) M Y^{t}\right) \cdots M Y^{t}\right) M Y^{t},
$$

which is given by $Q^{\prime}(t) / Q^{\prime \prime}(t)$, where

$$
\begin{aligned}
Q^{\prime}(t) & =2 t\left((1+z)-2\left(1+10 z+12 z^{2}\right) t-4 z(3+4 z)(2-13 z) t^{2}\right. \\
& \left.+64 z^{2}\left(2-3 z-12 z^{2}\right) t^{3}-256 z^{4}(1-4 z) t^{4}\right) \\
Q^{\prime \prime}(t) & =\left(1-(1+20 z) t-16 z(1-8 z) t^{2}+64 z^{2}(1-4 z) t^{3}\right) . \\
& \cdot\left(1-2(1+8 z) t-32 z(1-2 z) t^{2}\right) .
\end{aligned}
$$

This genus polynomial corresponds to the sequence of cubic Halin graphs illustrated in Figure 10. 


\section{Appendix A. Log-concavity proof for Example 4.5}

Theorem A.1. The genus polynomial as defined in Example 4.5 is logconcave.

Proof. Let $g_{n}(j)$ be defined as in (14). By direct calculations, with the help of mathematical programming, we can see that the function $g_{n}(j)$ can be expressed as

$$
\begin{aligned}
g_{n}^{\prime}(j) & =\left(g_{n}(j)\right)^{2}-g_{n}(j-1) g_{n}(j+1) \\
& =\frac{8^{2 j-2} p_{n}(j)(n-2-j) !(n-1-j) !}{j !(j+1) !(n+4-2 j) !(n+6-2 j) !} \\
& =\frac{8^{2 j-2}\left(a_{n}(j)+b_{n}(j) 2^{n-2 j}+c_{n}(j) 2^{2 n-4 j}\right)(n-2-j) !(n-1-j) !}{j !(j+1) !(n+5-2 j) !(n+7-2 j) !}
\end{aligned}
$$

for all $j=0,1,2, \ldots, \frac{n+4}{2}$. Fix $n_{0}=5$. (It is not hard to see that $g_{n}(z)$ is a log-concave polynomial, for $n=0,1, \ldots, n_{0}-1$.) Note that

$$
\begin{aligned}
p_{n}\left(\frac{n+4}{2}\right) & =0 \\
p_{n}\left(\frac{n+3}{2}\right) & =\frac{3}{2048}(n-5)\left(n^{2}-1\right)^{2}\left(n^{2}-9\right)^{2}(n+5)^{3} \\
p_{n}\left(\frac{n+2}{2}\right) & =\frac{3}{1024} n^{2}\left(n^{2}-16\right)\left(n^{2}-4\right)^{2}\left(3 n^{2}+34 n+104\right)^{2} \\
p_{n}\left(\frac{n+1}{2}\right) & =\frac{1}{2048}\left(n^{2}-3\right)\left(n^{2}-1\right)^{2}\left(397 n^{6}+13980 n^{5}+191365 n^{4}\right. \\
& \left.+1300200 n^{3}+5232943 n^{2}+11254140 n+14170815\right), \\
p_{n}\left(\frac{n}{2}\right) & =\frac{3}{2048} n^{2}\left(n^{2}-4\right)\left(747 n^{8}+35544 n^{7}+635960 n^{6}+5330304 n^{5}\right. \\
& +27585968 n^{4}+96775296 n^{3}+207246080 n^{2}+328052736 n \\
& +424673280), \\
p_{n}(0) & =256(n-1)(n+6)(n+5)(n+4)^{2}(n+3)^{2}(n+2)^{2}(n+1)^{2} n^{2},
\end{aligned}
$$

which shows that $g_{n}^{\prime}(j) \geq 0$, for all $j=0, n / 2, \ldots, \frac{n+4}{2}$, where $n \geq n_{0}$. Thus, it remains to show that $p_{n}(j) \geq 0$ for all $n \geq n_{0}$ and $j=j_{0}, j_{0}+1, \ldots, \frac{n-m}{2}$, where $j_{0}=1$ and $m=1$. By help of mathematical programming, we have 


$$
\begin{aligned}
& \frac{c_{n}(j)}{64 j(j+1)} \\
& =2 j(j+1)\left(44 j^{8}+1496 j^{7}+19099 j^{6}+108761 j^{5}+272641 j^{4}+223379 j^{3}\right. \\
& \left.+91976 j^{2}-70836 j-41760\right)+\left(16 j^{10}+1616 j^{9}+38136 j^{8}+372814 j^{7}\right. \\
& +1694724 j^{6}+3580360 j^{5}+3479084 j^{4}+1362866 j^{3}-519000 j^{2}-549816 j \\
& -83520)(n-2 j-1)+\left(192 j^{9}+10986 j^{8}+186496 j^{7}+1333805 j^{6}\right. \\
& +4356455 j^{5}+6563369 j^{4}+4379461 j^{3}+350132 j^{2}-1071664 j \\
& -324624)(n-2 j-1)^{2}+\left(984 j^{8}+38618 j^{7}+477683 j^{6}+2465405 j^{5}\right. \\
& \left.+5699415 j^{4}+5896237 j^{3}+2059190 j^{2}-795164 j-510384\right)(n-2 j-1)^{3} \\
& +\left(2816 j^{7}+79327 j^{6}+708717 j^{5}+2585905 j^{4}+4088631 j^{3}+2548087 j^{2}\right. \\
& -8383 j-413140)(n-2 j-1)^{4}+\left(4945 j^{6}+100227 j^{5}+633035 j^{4}\right. \\
& \left.+1576145 j^{3}+1562517 j^{2}+370267 j-175156\right)(n-2 j-1)^{5}+\left(5524 j^{5}\right. \\
& \left.+79080 j^{4}+340468 j^{3}+538758 j^{2}+263590 j-27396\right)(n-2 j-1)^{6} \\
& +\left(3946 j^{4}+38480 j^{3}+105812 j^{2}+90034 j+7740\right)(n-2 j-1)^{7}+\left(1768 j^{3}\right. \\
& \left.+11031 j^{2}+16901 j+4740\right)(n-2 j-1)^{8} \\
& +\left(473 j^{2}+1671 j+996\right)(n-2 j-1)^{9}+4(25+17 j)(n-2 j-1)^{10} \\
& +1 j^{2} j-11 .
\end{aligned}
$$

Note that each coefficient $(n-1-2 j)^{k}$ in $c_{n}(j)$ is non-negative (to see this, we expand it as a Taylor series at $j=1$ ) for all $j=j_{0}, j_{0}+1, \ldots, \frac{n-m}{2}$ and $n \geq n_{0}$, which implies that $c_{n}(j) \geq 0$. By considering the polynomial $b_{n}^{\prime}(j)=b_{n}(j)+2 c_{n}(j)(n-2 j)$, we can write

$$
\begin{aligned}
& \frac{b_{n}^{\prime}(j)}{2 j} \\
& =4 j(j+1)^{2}\left(2114 j^{8}+71731 j^{7}+925904 j^{6}+5579116 j^{5}+17427686 j^{4}\right. \\
& \left.+32050309 j^{3}+43226256 j^{2}+9744324 j-11049840\right)+(j+1)\left(10082 j^{10}\right. \\
& +454321 j^{9}+7680439 j^{8}+61907776 j^{7}+262181550 j^{6}+645130555 j^{5}
\end{aligned}
$$




$$
\begin{aligned}
& +1119325261 j^{4}+1174012884 j^{3}+367290188 j^{2}-182491296 j \\
& -49544640)(n-2 j-1)+\left(1554 j^{11}+176992 j^{10}+4935781 j^{9}\right. \\
& +59186354 j^{8}+358814772 j^{7}+1221679428 j^{6}+2695500871 j^{5} \\
& +4142206876 j^{4}+3806547986 j^{3}+1327933310 j^{2}-336379012 j \\
& -201485808)(n-2 j-1)^{2}+\left(18657 j^{10}+1179722 j^{9}+22969084 j^{8}\right. \\
& +198950098 j^{7}+886460794 j^{6}+2332944710 j^{5}+4149168772 j^{4} \\
& \left.+4798258248 j^{3}+2670254581 j^{2}+9055894 j-335614096\right)(n-2 j-1)^{3} \\
& +\left(95517 j^{9}+4102990 j^{8}+57791208 j^{7}+366417456 j^{6}+1231628848 j^{5}\right. \\
& +2585411604 j^{4}+3623269603 j^{3}+2789382330 j^{2}+509187784 j \\
& -290774124)(n-2 j-1)^{4}+\left(272217 j^{8}+8360350 j^{7}+85575280 j^{6}\right. \\
& +399853916 j^{5}+1040794390 j^{4}+1756122824 j^{3}+1767468877 j^{2} \\
& +626324782 j-130173756)(n-2 j-1)^{5}+\left(473154 j^{7}+10454268 j^{6}\right. \\
& +\left(37270344 j^{5}+269375610 j^{4}+558835786 j^{3}+720028758 j^{2}\right. \\
& \left.+32398334 j^{2}+36637976 j 9011484\right)(n-2 j-1)^{8}+\left(145113 j^{4}\right. \\
& +394308268 j-15248716)(n-2 j-1)^{6}+\left(517065 j^{6}+8103196 j^{5}\right. \\
& +43007904 j^{4}+115604554 j^{3}+191125107 j^{2}+151161034 j \\
& +14704212)(n-2 j-1)^{7}+\left(353319 j^{5}+3832486 j^{4}+14871169 j^{3}\right. \\
& + \\
& \left.+1072206 j^{3}+3300683 j^{2}+5503362 j+2483116\right)(n-2 j-1)^{9} \\
& +
\end{aligned}
$$

Note here that each coefficient $(n-1-2 j)^{k}$ in $b_{n}^{\prime}(j)$ is non-negative (again, we need only expand it as a Taylor series at $j=1$ ) for all $j=$ $j_{0}, j_{0}+1, \ldots, \frac{n-m}{2}$ and $n \geq n_{0}$, which implies that $b_{n}^{\prime}(j) \geq 0$. By considering the polynomial

$$
b_{n}^{\prime \prime}(j)=a_{n}(j)+2 b_{n}(j)(n-2 j)+4 c_{n}(j)(n-2 j)^{2}
$$


we can write

$$
\begin{aligned}
& \frac{b_{n}^{\prime \prime}(j)}{2 j} \\
& =4 j(j+1)^{2}\left(2114 j^{8}+71731 j^{7}+925904 j^{6}+5579116 j^{5}+17427686 j^{4}\right. \\
& \left.+32050309 j^{3}+43226256 j^{2}+9744324 j-11049840\right)+(j+1)\left(10082 j^{10}\right. \\
& +454321 j^{9}+7680439 j^{8}+61907776 j^{7}+262181550 j^{6}+645130555 j^{5} \\
& +1119325261 j^{4}+1174012884 j^{3}+367290188 j^{2}-182491296 j \\
& -49544640)(n-2 j-1)+\left(1554 j^{11}+176992 j^{10}+4935781 j^{9}\right. \\
& +59186354 j^{8}+358814772 j^{7}+1221679428 j^{6}+2695500871 j^{5} \\
& +4142206876 j^{4}+3806547986 j^{3}+1327933310 j^{2}-336379012 j \\
& -201485808)(n-2 j-1)^{2}+\left(18657 j^{10}+1179722 j^{9}+22969084 j^{8}\right. \\
& +198950098 j^{7}+886460794 j^{6}+2332944710 j^{5}+4149168772 j^{4} \\
& \left.+4798258248 j^{3}+2670254581 j^{2}+9055894 j-335614096\right)(n-2 j-1)^{3} \\
& +\left(95517 j^{9}+4102990 j^{8}+57791208 j^{7}+366417456 j^{6}\right. \\
& +1231628848 j^{5}+2585411604 j^{4}+3623269603 j^{3}+2789382330 j^{2} \\
& -290774124+509187784 j)(n-2 j-1)^{4}+\left(272217 j^{8}+8360350 j^{7}\right. \\
& +85575280 j^{6}+399853916 j^{5}+1040794390 j^{4}+1756122824 j^{3} \\
& \left.+1767468877 j^{2}+626324782 j-130173756\right)(n-2 j-1)^{5} \\
& +\left(473154 j^{7}+10454268 j^{6}+77270344 j^{5}+269375610 j^{4}\right. \\
& \left.+558835786 j^{3}+720028758 j^{2}+394308268 j-15248716\right)(n-2 j-1)^{6} \\
& +\left(517065 j^{6}+8103196 j^{5}+43007904 j^{4}+115604554 j^{3}\right. \\
& \left.+191125107 j^{2}+151161034 j+14704212\right)(n-2 j-1)^{7} \\
& +\left(353319 j^{5}+3832486 j^{4}+14871169 j^{3}+32398334 j^{2}\right. \\
& +36637976 j+9011484)(n-2 j-1)^{8}+\left(145113 j^{4}+1072206 j^{3}\right. \\
& \left.+3300683 j^{2}+5503362 j+2483116\right)(n-2 j-1)^{9}+\left(32808 j^{3}\right.
\end{aligned}
$$




$$
\begin{aligned}
& \left.+176084 j^{2}+471768 j+382764\right)(n-2 j-1)^{10}+\left(3396 j^{2}\right. \\
& +18384 j+31884)(n-2 j-1)^{11}+(96 j+1120)(n-2 j-1)^{12} .
\end{aligned}
$$

Note here, too, that each coefficient $(n-1-2 j)^{k}$ in $b_{n}^{\prime \prime}(j)$ is non-negative (once again, we need only expand it as a Taylor series at $j=1$ ) for all $j=j_{0}, j_{0}+1, \ldots, \frac{n-m}{2}$ and $n \geq n_{0}$, which implies that $b_{n}^{\prime \prime}(j) \geq 0$. Therefore, by Theorem 5.1, the polynomial $g_{n}^{\prime}(j) \geq 0$ for all $j=j_{0}, j_{0}+1, \ldots, \frac{n-m}{2}$ and $n \geq 5$. It follows that the polynomial $g_{n}(z)$ is log-concave.

\section{References}

[1] J. L. Gross (2011). Genus distribution of graph amalgamations: selfpasting at root-vertices. Australasian J. Combin. 49 19-38. MR2790958

[2] J. L. Gross (2013). Embeddings of cubic Halin graphs: genus distributions, Ars Math. Contemporanea 6 37-56. MR2928443

[3] J. L. Gross (2014). Embeddings of graphs of fixed treewidth and bounded degree, Ars Math. Contemporanea 7 379-403. Presented at the AMS Meeting of January, 2012, in Boston, MA.

[4] J. L. Gross, I. F. Khan and M. I. Poshni (2010). Genus of graph amalgamations: pasting at root-vertices, Ars Combinatoria 94 33-53. MR2599716

[5] J. L. Gross, T. Mansour, T. W. Tucker and D. G. L. Wang (2013). Iterated claws have real-rooted genus polynomials. Presented (JG) as keynote paper at GEMS 2013 in Slovakia. Conference proceedings to appear in Ars Math. Contemporanea.

[6] J. L. Gross, T. Mansour and T. W. Tucker (2014). Log-concavity of genus distributions of ring-like families of graphs. European J. Combin. 42 74-91.

[7] J. L. Gross, T. Mansour, T. W. Tucker and D. G. L. Wang (2013). Logconcavity of the genus polynomials of Ringel ladders.

[8] I. F. Khan, M. I. Poshni and J. L. Gross (2010) Genus distribution of graph amalgamations at roots of higher degree. Ars Math. Contemporanea 3 121-138. MR2718143

[9] M. I. Poshni, I. F. Khan and J. L. Gross (2010). Genus distribution of edge-amalgamations, Ars Math. Contemporanea 3 69-86. MR2606579 
Jonathan L. Gross

Department of Computer Science

Columbia University

NEW YoRK, NY 10027

USA

E-mail address: gross@cs.columbia.edu

TOUFIK MANSOUR

Department of Mathematics

UNIVERSITY OF HAIFA

3498838 HAIFA

ISRAEL

E-mail address: tmansour@univ.haifa.ac.il

Thomas W. Tucker

Department of Mathematics

Colgate University

HAMILTON, NY 13346

USA

E-mail address: ttucker@colgate.edu

Received December 13, 2013 